\title{
El régimen fiscal cooperativo como instrumento de política económica*
}

\author{
Juan Calvo Vérgez \\ Profesor Titular de Derecho Financiero y Tributario \\ Universidad de Extremadura
}

Sumario: I. Introducción. II. La importancia del papel desempeñado por las cooperativas de trabajo asociado, las cooperativas de viviendas y las cooperativas de consumidores y usuarios. III. Creación de empleo y reducción de la carga tributaria derivada de la fiscalidad de las cooperativas. IV. La actual insuficiencia de la fiscalidad favorable como motor del desarrollo cooperativo a la vista de los mecanismos de promoción existentes. V. El conocimiento del cooperativismo como primer paso de intervención y la necesidad de mejorar la financiación de los socios y de las cooperativas. VI. Reflexiones finales acerca de la formación y difusión del cooperativismo.

Resumen: El presente estudio tiene por objeto analizar la trascendencia que en la actualidad adquiere el régimen fiscal cooperativo como instrumento destinado a reforzar el papel de las cooperativas en el desarrollo de la actividad económica. Dicho régimen se revela en la actualidad como insuficiente, no habiéndose aprovechado la reciente Reforma Tributaria para reforzar el mismo. Y ello sin perjuicio de reconocer la trascendencia del papel desempeñado hasta la fecha por las distintas modalidades de sociedades cooperativas en los ámbitos relativos a la creación de empleo y al desarrollo tejido empresarial. Asimismo se subrayan otras actuaciones pendientes tales como la necesidad de mejorar la financiación de los socios y, en definitiva, reforzar la formación y difusión del cooperativismo.

Palabras clave: Cooperativas, régimen fiscal, impuestos directos e indirectos, socios, cooperativismo, financiación, difusión.

Abstract: This study aims to examine the importance that the cooperative tax scheme has today as an instrument to strengthen the role of cooperatives in the development of economic activity. This scheme proves to be inad-

* El presente artículo tiene su origen en una Comunicación presentada a las pasadas "Jornadas sobre entidades con valor social: nuevas perspectivas tributarias», celebradas el 12 de febrero de 2015 en la Sede del Instituto de Estudios Fiscales, Madrid. 
equate at present, as no advantage has been taken of the recent Tax Reform to strengthen it. And this while recognising the significant role played so far by the various forms of cooperative societies in areas related to job creation and business development. Other actions still to be undertaken are also highlighted such as the need to improve the financing of cooperative members and, ultimately, enhance training and the dissemination of cooperativism.

Key words: Cooperatives, tax scheme, direct and indirect taxes, members, cooperativism, financing, dissemination. 


\section{Introducción}

Como es sabido las técnicas de realización de políticas sociales actualmente existentes no se limitan únicamente al gasto público y al disfrute, en el ámbito estrictamente tributario, de la aplicación de un conjunto de exenciones. Por el contrario cabe aludir al surgimiento de un amplio campo que el ordenamiento jurídico ha reservado a la iniciativa privada y que se apoya en la presencia de una norma jurídica que delimita las situaciones y la legitimidad para actuar así como, en la mayor parte de las ocasiones, en la existencia de un conjunto de subvenciones de carácter finalista. Y todo ello al margen de la importancia que adquiere en el presente caso el esfuerzo personal y financiero de los interesados.

La importancia de estas figuras y técnicas para la realización de políticas sociales se encuentra fuera de toda duda. Su agrupación y presentación se lleva a cabo bajo el rótulo genérico de entidades de economía social. Sus tipos jurídicos son, principalmente, cooperativas, sociedades laborales, fundaciones, mutualidades de previsión social, asociaciones de utilidad pública, empresas de inclusión social y centros especiales de empleo. Los rasgos esenciales de este conjunto de actividades y de entidades pueden reducirse a dos. En primer lugar, que desembocan directamente en la producción de bienes y servicios con una importancia escasa de lo que podríamos denominar como economía financiera. Estas entidades no tienen acceso a los mercados de capitales, a diferencia de las empresas capitalistas. Más aún, sus dificultades financieras son mayores. Su fuerza está, en consecuencia, en la aportación de trabajo, en el estímulo que supone la participación y en la ilusión importante que aportan sus diferentes trabajadores. Y, en segundo término, su campo de actuación presenta un carácter amplio, lo que contribuye a desbordar la producción de bienes y servicios requerida para actuar en el ámbito del consumo (así sucede, por ejemplo, con las cooperativas de consumidores y usuarios y de vivienda1) y la propia preparación requerida para el empleo (en el caso de las empresas de inclusión social y centros especiales de empleo).

Con carácter general la Constitución Española (CE) establece una norma de apoyo a las empresas asociativas (que representan el gran núcleo de estas entidades) y que, a nuestro juicio, no ha tenido una

1 Véase a este respecto GADEA SOLER, E., «Cooperativismo de consumo: la adaptación del modelo a las nuevas condiciones del mercado», Boletín de la Asociación Internacional de Derecho Cooperativo (Journal International Association of Cooperative Law), núm. 45, 2011, págs. 191 y ss. 
atención suficiente por parte del Estado. Dice su art. 129.2 que «Los poderes públicos (...) fomentarán, mediante una legislación adecuada, las sociedades cooperativas. También establecerán los medios que faciliten el acceso de los trabajadores a la propiedad de los medios de producción». Por su parte el párrafo segundo de este art. 129 sí que ofrece un contenido social directo, motivo por el cual resulta difícil de entender su ubicación fuera del Capítulo de la Norma Fundamental referido a los principios sociales y económicos. Tal y como se afirma en dicho párrafo «Los poderes públicos promoverán eficazmente las diversas formas de participación en la empresa y fomentarán, mediante una legislación adecuada, las sociedades cooperativas. También establecerán los medios que faciliten el acceso de los trabajadores a la propiedad de los medios de producción». Se trata, por tanto, de un precepto que tiene los dos componentes contenidos en el Capítulo III de nuestra Carta Magna: mandato a los poderes públicos y contenido social. Incluso los mecanismos de promoción previstos para cada caso se encuentran establecidos y regulados en nuestro ordenamiento de manera específica: sociedades cooperativas, mutualidades y sociedades laborales.

\section{La importancia del papel desempeñado por las cooperativas de trabajo asociado, las cooperativas de viviendas y las cooperaitvas de consumidores y usuarios}

Sin lugar a dudas, al desarrollo de políticas sociales de creación de empleo estable y de mayor participación de los trabajadores sirven, entre otras, las llamadas cooperativas de trabajo asociado, caracterizadas por la existencia de un funcionamiento democrático (su régimen es de libre adhesión y baja voluntaria) y por la presencia de un régimen de derechos y de participación de los socios muy amplio. Estas características contribuyen a que la aproximación de los trabajadores a la toma de decisiones en la empresa sea máxima, con un aumento de la productividad, responsabilidad y estabilidad de los trabajadores mismos, lo que constituye un hecho muy positivo que se inscribe en la mejor política de empleo posible.

Las cooperativas de trabajo asociado participan de la tradición y el prestigio del mundo cooperativo. Su referencia moderna se inscribe en el desarrollo del cooperativismo en las últimas décadas del Siglo XIX. Posteriormente la Ley de 4 de julio de 1931 de la Segunda República, sobre la delimitación de este tipo de sociedad, contribuyó a fijar sus condiciones legales. Especial trascendencia adquiere a este respecto la Constitución de 1978 y, en particular, el mandato contenido en su 
art. 129.2, a cuyo tenor «Los Poderes Públicos (...) fomentarán mediante una legislación adecuada las sociedades cooperativas».

Actualmente, como es bien sabido, el cooperativismo se rige por una Ley general de 16 de julio de 1999 así como por diversas leyes especiales en casi todas las Comunidades Autónomas². Su prestigio económico y social está fuera de toda duda apoyado en su funcionamiento democrático, en el principio de libre adhesión y baja voluntaria y en un grado de participación de los socios muy extenso. Se puede decir, por tanto, que los trabajadores han hecho honor a esta confianza del legislador con una participación responsable y han constituido un mecanismo de arraigo de estos mismos trabajadores en su territorio, lo que resulta particularmente significativo en un momento como el actual, caracterizado por la existencia de una progresiva deslocalización empresarial que incide negativamente sobre el empleo y su estabilidad ${ }^{3}$. En definitiva, las cooperativas de trabajo asociado han cumplido su función de alternativa a la empresa capitalista en cuanto a la creación de empleo estable y participativo se refiere.

A priori, los mecanismos de actuación de los poderes públicos en el fomento que ordena la Constitución siguen dos caminos diferentes. Primero, las subvenciones de la Administración General y de las Administraciones autonómicas con ocasión de situaciones diversas: creación, ampliación de puestos de trabajo, formación, inversiones y acciones de mejora de la competitividad, etc. La segunda vía es un régimen fiscal específico más favorable que el normal y cuyo principal exponente es una tributación bonificada en el Impuesto sobre Sociedades. Y, a este respecto, la Ley 20/1990, de 19 de diciembre, de Régimen Fiscal de las Cooperativas, distingue entre cooperativas protegidas (con un tipo de gravamen reducido) y especialmente protegidas (con una bonificación, además, del 50 por 100 de la cuota íntegra). ${ }^{4}$

2 Un minucioso estudio de la reciente regulación aprobada, por ejemplo, en la Comunidad Autónoma de Cantabria puede encontrarse en GADEA SOLER, E. y DÍAZ ÁCIMAS, L. A., Régimen jurídico de las cooperativas de Cantabria: análisis de la Ley 6/2013, de 6 de noviembre, Dykinson, Madrid, 2014.

3 Dice MONZÓN, J. L., que «En el actual contexto de globalización, donde los procesos de deslocalización de actividades productivas desafían constantemente a los territorios, los actores de la Economía Social sitúan en la sociedad civil las riendas del proceso económico (...) generando un mayor anclaje de las empresas y una mayor autonomía de los territorios para definir su propio modelo de desarrollo» («Economía Social y Sociedad de Bienestar: actores y ámbitos de actuación en España», en Anuario de la Economía Social 2007-2008, Cepes, Madrid, 2008, pág. 44).

4 Las cooperativas especialmente protegidas son de trabajo asociado, agrarias, explotación comunitaria de la tierra y del mar. Son de personas físicas y están sometidas a determinados límites de diversa naturaleza, aunque orientados a una primacía de las 
El contrapunto de la promoción estatal a las cooperativas no es solo la fidelidad a su naturaleza (funcionamiento democrático y, por ello, participación importante de los trabajadores). Es también la exigencia de un grado de solvencia específico a través del Fondo de Reserva obligatorio e irrepartible entre los socios que la cooperativa debe constituir con independencia de los que se establezcan con carácter imperativo en función de su actividad o calificación. Igualmente, la constitución, también obligatoria, de un Fondo de Educación y Promoción destinado principalmente a la difusión del cooperativismo, a la promoción de las relaciones intercooperativas y al desarrollo profesional. En definitiva, una dotación que busca el fortalecimiento del espíritu cooperativo, las acciones asociativas y la promoción profesional de los socios. Objetivos, sin duda, del mayor interés y que han contribuido a un fortalecimiento de este tipo de empresa a que nos referimos.

Adicionalmente, en torno a la presente figura de empresa asociativa se han ido configurando diversos tipos de apoyo que presentan un interés innegable. El primero de ellos se concreta en la posibilidad de que las prestaciones de desempleo a favor de los trabajadores puedan acumularse y percibirse de una sola vez, lo que facilita que este importe sea destinado a la creación de una empresa asociativa (cooperativa de trabajo asociado o sociedad laboral). Se trata de una acumulación que facilita esta constitución, como acabamos de decir, y que supone una ventaja financiera para la Administración, que pasa a percibir una vez creada la sociedad de que se trate los tributos que se deriven de la actividad social correspondiente y que compensa ampliamente la desventaja financiera de adelantar el pago de las prestaciones.

cualidades personales sobre los elementos económicos. Por otra parte, los beneficios fiscales no se limitan al Impuesto sobre Sociedades. Se extienden, también, a los Impuestos sobre Transmisiones Patrimoniales y Actos Jurídicos Documentados, sobre Actividades Económicas o Impuesto sobre Bienes Inmuebles (bienes de naturaleza rústica de las Cooperativas Agrarias y de Explotación Comunitaria de la Tierra). Un estudio de los motivos que han llevado al legislador al establecimiento de un régimen fiscal favorable para este tipo de entidades puede verse en DE LUIS ESTEBAN, J. M., "Reflexiones sobre el futuro fiscal de las cooperativas» y especialmente en el capítulo "La función social como contrapartida», en La Fiscalidad de las Entidades de Economía Social (Dir.: Calvo Ortega, R.), Thomson-Civitas, Madrid, 2005, págs. 189 y ss. Véanse igualmente a este respecto COSÍN OCHAITA, R., "Régimen de cooperativas y regímenes especiales», Carta Tributaria, núm. 2, 2006, págs. 7 y ss., CRESPO MIEGIMOLLE, M., Régimen especial de las sociedades cooperativas en el nuevo Impuesto sobre Sociedades, Comares, Granada, 1998, págs. 75 y ss. y MARTíN FERNÁNDEZ, J., MARTíN SALCINES, F. y RODRÍGUEZ MÁRQUEZ, J., Cuestiones tributarias y contables de las cooperativas, lustel, 2006, págs. 52 y ss. 
La segunda medida de apoyo es la exención del Impuesto sobre la Renta de las Personas Físicas de esta prestación de desempleo acumulada. De conformidad con lo dispuesto por la letra n) del art. 7 de la Ley 35/2006, de 28 de noviembre, reguladora del IRPF5, quedan exoneradas de gravamen las prestaciones por desempleo reconocidas por la respectiva entidad gestora cuando se perciban en la modalidad de pago único establecida en el Real Decreto 1044/1985, de 19 de junio, por el que se regula el abono de la prestación por desempleo en su modalidad de pago único, siempre que las cantidades percibidas se destinen a las finalidades y en los casos previstos en la citada norma, hallándose además condicionada la aplicación de la citada exención al mantenimiento de la acción o participación durante el plazo de cinco años (en el supuesto de que el contribuyente se hubiera integrado en sociedades laborales o cooperativas de trabajo asociado o hubiera realizado una aportación al capital social de una entidad mercantil) o bien al mantenimiento, durante idéntico plazo, de la actividad, en el caso del trabajador autónomo.

En resumen, las cooperativas de trabajo asociado representan una figura a través de la cual tiene lugar la integración y realización del Estado Social. Y ello debido a que contribuyen tanto a la realización de una política social como a la creación de un empleo estable y participativo que se basa, a su vez, en la aproximación de los trabajadores a la toma de decisiones en la empresa. Una política social que busca la realización del principio de igualdad, si bien en el presente caso en lo que se refiere al ejercicio de una actividad empresarial en forma asociativa.

Por otra parte, todo análisis que pretenda efectuarse de la realización de políticas sociales relacionadas esencialmente con el consumo y caracterizadas por el hecho de que su ejecución es llevada a cabo directamente por los propios interesados ha de referirse necesariamente a las cooperativas de viviendas y a las cooperativas de consumidores y usuarios.

Con carácter general el Capítulo III, Título I de la Constitución establece, entre otros, dos mandatos concretos que constituyen dos políticas sociales determinadas: el acceso de los ciudadanos a una vivienda digna y adecuada y el fomento de la defensa de los consumidores a través de su propia organización. Más concretamente declara la Ley

5 Como es sabido la actual redacción de dicha letra tiene su origen, con efectos desde el 1 de enero de 2013, en el número uno del art. 8 de la Ley 11/2013, de 26 de julio, de medidas de apoyo al emprendedor y de estímulo del crecimiento y de la creación de empleo. 
Fundamental en su art. 47, refiriéndose al primero de ellos, que «Todos los españoles tienen derecho a disfrutar de una vivienda digna y adecuada. Los poderes públicos promoverán las condiciones necesarias y establecerán las normas pertinentes para hacer efectivo este derecho (...)».

Pues bien en el presente caso nuestro ordenamiento jurídico, junto a medidas muy diversas de promoción del acceso a la vivienda (de carácter administrativo y fiscal, principalmente) utiliza la figura jurídica de la cooperativa para permitir que los propios interesados puedan realizar esta política. El mismo cuadro se repite en relación con los consumidores. Sin perjuicio de la existencia de numerosas normas y medidas de protección del consumo, la Constitución ordena a los poderes públicos que fomenten «sus organizaciones». También aquí concurren un conjunto de normas y de medidas diversas pero, además, se utilizan figuras y técnicas jurídicas ya establecidas por el ordenamiento para que los propios interesados puedan realizar estas políticas. Es la cooperativa de consumidores y usuarios.

La nota diferencial de estas entidades es que permiten que los propios ciudadanos realicen las políticas sociales que les interesan. Es cierto que esta doble vertiente se origina en algunas otras figuras de la Economía Social, pero aquí se da con una mayor nitidez y separación de otras finalidades.

Las cooperativas de viviendas buscan la realización por los interesados de la política de vivienda digna y adecuada establecida por el art. 47 de la Constitución antes citado. Sin entrar aquí a estudiar en profundidad este tipo cooperativo ${ }^{6}$, sí resulta obligado resaltar sus aspectos esenciales. Primero, su flexibilidad, que en la práctica constituye un requisito esencial para su éxito. Se permite la construcción de instalaciones distintas que resulten obligadas, urbanización de terrenos, enajenación o arrendamiento a terceros no socios de locales comerciales y edificaciones complementarias, construcciones por fases o promociones dotando a cada una de ellas de autonomía de gestión y patrimonial y contabilidad independiente, Juntas especiales de socios y, por citar un último ejemplo, la posibilidad de transmisión de derechos por los socios de la cooperativa dentro de unos requisitos lógicos y proporcionados. En definitiva, esta flexibilidad debe ser destacada porque constituye un elemento esencial para el progreso de esta técnica de acceso a la propiedad de la vivienda. Significa una adecuación a la reali-

6 Su regulación con carácter general se encuentra recogida en la Ley 27/1999, de 16 de junio, de Cooperativas. 
dad presente y a las futuras modificaciones que puedan presentarse, incluso la necesidad de transmisión de la vivienda misma. ${ }^{7}$

El segundo aspecto esencial de la cooperativa de viviendas es la seguridad jurídica del socio. También aquí la Ley 27/1999 citada incorpora mecanismos que contribuyen a este valor de la seguridad que se considera esencial. Entre otros, la exigencia de estatutos de la cooperativa, las medidas adicionales cuando la constitución se haga por fases o promociones, la prohibición de percepción de remuneraciones o compensaciones por el desempeño del cargo, la exigencia de autoría de cuentas en los casos que puedan presentar una mayor conflictividad y, en todo caso, cuando lo prevean los Estatutos o lo acuerde la Asamblea General.

Todo ello con independencia de la acción de la Administración General del Estado y, concretamente, de la calificación y registro de estas cooperativas y de la función inspectora que corresponde a la Inspección de Trabajo y Seguridad Social.

La contribución de estas cooperativas a la realización de la política social de vivienda establecida en la Constitución recibe el apoyo del ordenamiento jurídico en forma diversa. Así, la aplicación de un régimen fiscal especial (más favorable, lógicamente, que el normal) establecido en la Ley 20/1990, de 19 de diciembre, con exenciones y bonificaciones en el Impuesto sobre Transmisiones Patrimoniales y Actos Jurídicos Documentados (ITPyAJD), Impuesto sobre Sociedades (IS) e Impuesto sobre Actividades Económicas (IAE). Además, las subvenciones que puedan establecerse por las distintas Administraciones y cesiones de terrenos finalistas, entre otras medidas muy diversas.

En resumen, promover y favorecer el acceso a la vivienda a los ciudadanos es una política propia del Estado Social que tiene como peculiaridad ser gestionada por los propios administrados. Su importancia cuantitativa no puede desconocerse.

Por lo que respecta a las cooperativas de consumidores y usuarios, también aquí el punto de arranque está en la Constitución, como ya vimos, que ordena a los poderes públicos que fomenten las organizaciones de consumidores y usuarios.

7 La transmisión de derechos es una necesaria y prudente medida antibloqueo. El art. 92 de la Ley 27/1999 autoriza la transmisión de la vivienda pasados 5 años u otro plazo fijado por los Estatutos no superior a 10. Incluso antes de este plazo puede ponerla a disposición del Consejo Rector de la cooperativa, que puede ofrecerlos a los solicitantes de admisión como socios por orden de antigüedad y, a falta de solicitantes, puede transmitirlos inter vivos a terceros no socios. 
El esquema es paralelo al que acabamos de ver en relación con la vivienda. Estos poderes pueden utilizar, y lo hacen, distintas técnicas y medidas de protección al consumo; pero permiten y apoyan que los propios consumidores y usuarios establezcan sus propias organizaciones gestionadas por ellos mismos.

Así la Ley 27/1999, ya citada, regula las cooperativas de consumidores y usuarios en su art. 88. Aquí el legislador ha cuidado la amplitud del objeto de estas sociedades, al disponer que «El suministro de bienes y servicios adquiridos a terceros o producidos por la misma cooperativa para uso o consumo de los socios y de quienes con ellos conviven». Amplitud, pues, en el origen de los bienes (adquiridos o de producción propia) ${ }^{8}$. También una tensión importante en la atribución de la cualidad de socio que resulta ampliada, de acuerdo con lo establecido en el citado art. 88, "a las personas físicas y las entidades $u$ organizaciones que tengan el carácter de destinatarios finales». Se extiende, pues, el concepto de consumidor o usuario a los destinatarios finales, que pueden ser sujetos no personas físicas (entidades u organizaciones).

Esta extensión alcanza su expresión más nítida con el precepto del art. 88 citado, según el cual «Las cooperativas de consumidores y usuarios podrán realizar operaciones cooperativizadas con terceros no socios, dentro de su ámbito territorial, si así lo prevén sus Estatutos». ${ }^{9}$

Se trata de un régimen flexible que favorece a estas cooperativas y, por tanto, al consumo. Sus límites son el ámbito territorial dentro del cual opera la cooperativa y lo dispuesto en los estatutos que figuran en la escritura pública de constitución y que solo pueden ser modificados por la Asamblea General. En definitiva, un régimen de flexibilidad que favorece la actuación de estas cooperativas y, por ello, a los consumidores.

La promoción de esta figura se lleva a cabo a través de los dos mecanismos anteriormente apuntados, a saber, concesión de subvencio-

8 Véase, a este respecto, RODRIGO URÍA, AURELIO MENÉNDEZ y MERCEDES VÉRGEZ, Curso de Derecho Mercantil, vol. I, Civitas, Madrid, 1999, págs. 233 y ss.

${ }^{9}$ La división entre operaciones cooperativizadas y las que no tienen este carácter es importante. Aquéllas se definen como las que son propias, objetiva y subjetivamente, de la cooperativa. Estas operaciones cooperativizadas realizadas con terceros no socios deben ser objeto de contabilización separada según la Disposición Adicional Sexta de la Ley 27/1999. Por otra parte la Ley 20/1990, de Régimen Fiscal de las Cooperativas, dispone en su artículo 13.10 como causa de la pérdida de la condición de cooperativa fiscalmente protegida «la realización de operaciones cooperativizadas con terceros no socios, fuera de los casos permitidos en las leyes, así como el incumplimiento de las normas sobre contabilización separada de tales operaciones y destino al Fondo de Reserva obligatorio de los resultados obtenidos en su realización». 
nes por las Administraciones estatal y autonómica y un régimen fiscal favorable. En este campo de la fiscalidad, las cooperativas de consumidores y usuarios se incluyen en el grupo de las cooperativas especialmente protegidas que es, como ya indicamos, el más favorable para estas sociedades en su consideración como contribuyentes, aunque limitado a aquellas de entidad más reducida. ${ }^{10}$

En resumen, este tipo de cooperativas, con las ayudas indicadas, realiza una política social concreta prevista en la Constitución: fomento de las organizaciones de consumidores. También aquí estamos en presencia de una política propia del Estado Social.

\section{Creación de empleo y reducción de la carga tributaria derivada de la fiscalidad de las cooperativas}

La creación de empleo constituye hoy uno de los grandes objetivos en las sociedades modernas. La insuficiencia del crecimiento de los puestos de trabajo (e incluso su disminución en etapas de crisis) ha llegado a constituir en bastantes países uno de los principales problemas sociales, económicos y políticos. En algunos países, como en España, el mayor. El establecimiento de exenciones, bonificaciones, tipos de gravamen reducidos, etc., constituye uno de los mecanismos utilizados tradicionalmente para fomentar la creación de empleo a que nos referimos.

Las cuestiones que plantea este método son diversas: ¿es más eficaz la disminución de la carga fiscal que el otorgamiento de subvenciones? Dentro del ámbito fiscal, ¿es mejor actuar sobre los beneficios que sobre la fiscalidad de la actividad y los elementos de la empresa? ¿Cómo se debe medir la relación beneficio fiscal con el incremento del empleo? Etc. Son preguntas que tienen un componente político pero principalmente técnico y administrativo. Es necesario medir el coste fiscal y compararlo con los resultados en materia de empleo, lo que no siempre es fácil, ya que en las decisiones empresariales pueden incidir otras variables. Más seguro parece operar razonablemente sobre situaciones, estructuras y efectos conocidos y verificar el resultado de la manera más científica posible.

10 El artículo 12 de la Ley 20/1990 lleva a esta conclusión. Solo se aplica esta calificación «a las cooperativas que asocien a personas físicas (...) que la media de las retribuciones totales de los socios de trabajo no supere un determinado límite (...) y que las ventas a personas asociadas no superen un porcentaje determinado en relación con las totales». 
El punto de partida de nuestro razonamiento es que a dimensiones paralelas las cooperativas de trabajo asociado emplean comparativamente menos capital que las empresas privadas, al menos en sus primeras fases de actuación. Utilizan más el factor trabajo por sus menores disponibilidades financieras y porque es el factor que dominan mejor sus empleados. En otras palabras, el factor trabajo es el primero en la mayoría de las cooperativas a que nos referimos. En consecuencia a iguales cantidades de desgravación fiscal el resultado en términos de empleo es inicialmente y en lo que podrían denominarse primeras etapas empresariales, más positivo; es decir, el coste fiscal del empleo sería menor.

El mismo razonamiento mutatis mutandis puede emplearse en las situaciones de crisis económica en la empresa y, en consecuencia, ante la necesidad de reducción de empleo. Las técnicas de reducción lineal o de rotación se implantan normalmente más fácilmente en una cooperativa de trabajo asociado que en una empresa capitalista privada. La mayor aproximación a la toma de decisiones que se da en la cooperativa contribuye a una mayor comprensión y flexibilización de la situación; y la misma contribución realiza el sentimiento de copropiedad («cosa propia») que se observa en las cooperativas y que coloca la salvación del puesto de trabajo por encima de cualquier otra solución.

Esta mayor intensidad del factor trabajo tiene otra consecuencia favorable: el mayor y más rápido retorno dinerario al Tesoro Público (a través de instituciones tales como la Seguridad Social y la propia Administración tributaria) derivado de los recursos generados por las cooperativas. Las cuotas fiscales (impuesto sobre los rendimientos del trabajo y cuotas de la Seguridad Social) son superiores y de formación más rápida que las que podrían derivarse de una mayor capitalización. A un mismo nivel de exenciones, bonificaciones y beneficios fiscales corresponde un mayor retorno y también un retorno más fácil.

\section{La actual insuficiencia de la fiscalidad favorable como motor del desarrollo cooperativo a la vista de los mecanismos de promoción existentes}

La fortaleza actual del mundo cooperativo es hoy desigual. Lo es por la diferente importancia y desarrollo que tiene cada tipo de ellas. Las Cooperativas Agrarias tienen una fortaleza que dice mucho en su favor aunque, como hemos dicho anteriormente, son cooperativas de empresarios que favorecen a la totalidad de éstos y cuyo interés convergente hace que su implantación y extensión esté asegurada. Las de 
Viviendas siguen una marcha cuantitativa dictada por el mercado inmobiliario, aunque hay que reconocer la bondad de su idea y el servicio que prestan y el mérito de una gestión difícil. Las Cooperativas de Consumidores y Usuarios sirven a una idea muy acertada, aunque su ámbito es reducido dada la presión cada día más intensa y las innovaciones constantes de los grandes oferentes de bienes de consumo y de prestadores de servicios.

Las Cooperativas de Trabajo Asociado, cuyo objeto es la producción y comercialización asociada de bienes y servicios para terceros ${ }^{11}$, reflejan mejor que cualquier otro tipo los efectos de las medidas de promoción y apoyo a las mismas. Los beneficios fiscales ya citados son evidentemente importantes. No obstante no han conseguido, salvo excepciones muy significativas y honrosas, el desarrollo que hay que esperar lógicamente de un modelo económico como el cooperativo, cuya gestión, participación y responsabilidad son muy positivos, como es bien sabido.

El modelo de beneficio fiscal se ha mostrado insuficiente, no obstante su importancia innegable, principalmente el relativo a las cooperativas especialmente protegidas. Es cierto que la representación previa de un tipo de gravamen sobre los beneficios futuros actúa como instrumento de promoción de la creación. Ahora bien, este efecto futuro no ha sido decisivo. En parte, porque actúa en un momento posterior a la creación y funcionamiento de la cooperativa misma. Y, en parte también, porque la fiscalidad sobre el beneficio empresarial sigue una línea de atenuación como incentivo. Los beneficios fiscales establecidos para las empresas de reducida dimensión (libertad de amortización y tipo de gravamen más bajo que el normal) han reducido el atractivo fiscal de las cooperativas.

¿Podrían aumentarse los beneficios fiscales concedidos a las cooperativas? Es difícil en estos tiempos en que se busca un mayor rendimiento fiscal después de avanzar en la reducción del gasto y de alcanzar un nivel de eficiencia mayor. En este sentido la nueva Ley 27/2014, de 27 de noviembre, del Impuesto sobre Sociedades, no ha realizado concesiones en este sentido. El clima actual hace que las reducciones del tipo de gravamen se apliquen a las pymes y, más en concreto, a las empresas de reducida dimensión, sean o no sociedades cooperativas. Podría avanzarse en la simplificación de la norma fiscal de las cooperativas, en la homogeneización de la renta imponible, en el tratamiento

11 Dice la Ley General de Cooperativas, art. 80, que «tienen por objeto proporcionar a sus socios puestos de trabajo mediante su esfuerzo personal y directo (...) a través de la organización en común de la producción o servicios para terceros». 
de los fondos obligatorios pero difícilmente en la reducción de la carga tributaria. Desde nuestro punto de vista cabría la posibilidad de replantearse toda la situación actual. Es cierto que las cooperativas de trabajo asociado han demostrado una mayor resistencia a la crisis económica y, específicamente, a la destrucción de empleo (lo que es sin duda un hecho importante), pero debería de darse un paso adelante teniendo en cuenta que dichas cooperativas de trabajo asociado deben ser y han sido una figura de reacción de los propios trabajadores ante situaciones de manifiesta insuficiencia del empleo y que su resultado ha sido tradicionalmente muy estimable.

\section{$V$. El conocimiento del cooperativismo como primer paso de intervención y la necesidad de mejorar la financiación de los socios y de las cooperativas}

Tal y como se ha apuntado al inicio del presente trabajo el cooperativismo tiene más de un siglo a sus espaldas, como ya indicamos anteriormente en este trabajo. Las cooperativas de consumo y las llamadas cooperativas obreras se sitúan a finales del siglo XIX, como ya indicamos ${ }^{12}$. De entonces a nuestros días el desarrollo ha sido importante pero probablemente menor del que debía esperarse en un país con una tasa de desempleo tradicionalmente alta. Resulta, pues, necesario conocer las grandes razones y los obstáculos existentes que impiden un mayor desarrollo de esta forma de empresa.

La idea y el modelo de sociedad cooperativa son, sin duda, conocidos por la sociedad española, como no podía ser por menos. No lo es, sin embargo, científicamente y por un sector ciudadano que pueda calificarse de general. Las encuestas de opinión sobre las soluciones individuales al desempleo ponen de manifiesto la preferencia de los desempleados por la empresa privada capitalista y la empresa pública, que son las que dicen conocer, y las muy escasas preferencias por las fórmulas empresariales de autoempleo. Aunque no sea una respuesta razonada es significativa y plantea la necesidad de conocer sus causas y adoptar las medidas adecuadas.

La primera, a nuestro juicio, es un mejor conocimiento de esta figura. La falta de enseñanza detallada que se da en los centros universitarios de Derecho, Ciencias Empresariales, Económicas y, por supuesto, en las Escuelas de Negocio, es bien conocida. No se puede apreciar lo

12 DEL ARCO ÁlVAREZ, J. L., Régimen fiscal de las cooperativas, 1969, págs. 31 y ss. 
que no se conoce y no puede compararse con otros modelos cuando de uno de ellos, el cooperativo, solo se tiene una idea aproximada ${ }^{13}$. La sociedad cooperativa tiene tres rasgos esenciales que la hacen atractiva. Primero, la limitación de la responsabilidad de los socios, algo importante y apreciado por los trabajadores que no comprometen su patrimonio personal. Es un rasgo común con otros tipos sociales (salvo la regular colectiva), pero muy estimado en el campo laboral por razones obvias. Segundo, la gestión de la sociedad es democrática, lo que constituye un hecho diferencial esencial. No es solo que el trabajador se aproxime a la toma de decisiones. Es que participa en ellas. Este funcionamiento democrático genera dos hechos positivos. En primer lugar, facilita al máximo la aportación de experiencia y de conocimiento por parte de los socios. Esta aportación de experiencia y conocimientos mejora la productividad y, en consecuencia, da una mayor estabilidad a la sociedad cooperativa y contribuye también en conjunción con otros factores a aumentar su competitividad. En tercer lugar, genera un sentimiento de propiedad en común que dota de una especial flexibilidad a los órganos sociales en situaciones de especial dificultad producidas por la necesidad de la movilidad funcional o territorial, reducción de empleo, etc. Las experiencias que se conocen en materia de reducción de empleo abonan esta flexibilidad y las mayores posibilidades de buscar soluciones desde la convergencia.

Esta necesidad de un mayor conocimiento de las estructuras y funcionamiento de las cooperativas se extiende más allá del campo estricto de la enseñanza normalizada. Debe extenderse a campos tan importantes como el asesoramiento, la intervención, los servicios de empleo que son requeridos por los ciudadanos para la búsqueda de soluciones, entidades financieras, etc. En definitiva, un mayor y más profundo conocimiento de las Cooperativas de Trabajo Asociado por todos los actores que se relacionan con los demandantes de empleo conduce siempre a un mejor servicio profesional o público.

En la actualidad la cuestión relativa a la financiación de los socios y de la sociedad cooperativa se presenta, muy probablemente, como el problema de más difícil solución que tienen planteadas las Cooperativas de Trabajo Asociado. En este difícil aspecto hay que distinguir la financiación de los socios y la de la sociedad misma. En cuanto a la pri-

13 Acerca de esta cuestión véase GADEA SOLER, E., «Universidad y cooperativismo. Delimitación del concepto de cooperativa en una sociedad democrática avanzada: referencia a los principios cooperativos y a su discutida vigencia», Boletín de la Asociación Internacional de Derecho Cooperativo (Journal International Association of Cooperative Law), núm. 42, 2008, págs. 37 y ss. 
mera, las peculiaridades derivadas de la condición de Economía Social de las Cooperativas de Trabajo Asociado han llevado a dos formas de financiación que deben destacarse. La primera es la capitalización de la prestación de desempleo, que permite a los perceptores de la misma realizar una aportación estimable cuantitativamente a la cooperativa de la que pasan a formar parte. Tal y como ya se ha precisado esta capitalización ha sido reforzada con la exención de su percepción en el Impuesto sobre la Renta de las Personas Físicas, sometida a diversos requisitos, principalmente que no se exceda de un determinado límite y que la participación en la cooperativa se mantenga durante el plazo de cinco años ${ }^{14}$. En definitiva, la capitalización desnaturaliza en cierta medida la prestación de desempleo pero lo hace en la mejor dirección posible que he defendido en varias ocasiones y también ahora: sustituir financiación de desempleo por financiación de empleo. Lo que significa contribuir a la generación de un retorno financiero derivado de la nueva actividad económica que se inicia o apoya con la prestación capitalizada. En definitiva, la capitalización beneficia también al Tesoro Público, que comienza en plazo breve a recibir los retornos citados, a los que nos referiremos con detalle posteriormente.

La segunda forma de financiación específica son las subvenciones concedidas a estas Cooperativas de Trabajo Asociado (y a las sociedades laborales). La concesión la realizan normalmente las Comunidades Autónomas ligada a la creación de puestos de trabajo en la cooperativa de que se trate. Estas subvenciones son también del mayor interés para la economía en general y para las Comunidades Autónomas en particular. Para estas entidades, porque la actividad económica de que se trate se enraíza de modo particular en la Comunidad. En efecto, como ya indicamos las cooperativas que examinamos utilizan más intensamente factor trabajo, cuya volatilidad es inferior al factor capital. Este mayor enraizamiento favorece a la Comunidad, como es lógico. Además es perceptora directa de una parte de los retornos (o, si se quiere, de la recaudación impositiva que produce). De una manera directa porque el 50 por 100 del IRPF (y, en consecuencia, de las rentas del tra-

14 Recuérdese a este respecto que la Ley 11/2013, de 26 de julio, de Medidas de Apoyo al Emprendedor y de Estímulo al Crecimiento y de la Creación de Empleo, amplió las condiciones para la capitalización a que nos referimos. En relación con las cooperativas dispone, en su art. 4.5. ${ }^{a}$, que «la solicitud del abono de la prestación por desempleo de nivel contributivo (...) en todo caso deberá de ser de fecha anterior a la fecha de incorporación a la cooperativa o sociedad laboral (...) considerando que tal inicio coincide con la fecha que como tal figura en la solicitud de alta del trabajador de la Seguridad Social». 
bajo) se atribuyen a la Comunidad de que se trate; $y$, de una manera indirecta, porque igual atribución se hace del 50 por 100 del Impuesto sobre el Valor Añadido (IVA) y del 58 por 100 de los Impuestos Especiales (II.EE.). Y este consumo depende, en gran parte, de la actividad económica en el territorio de que se trate. Las subvenciones finalistas condicionadas benefician también a la economía en general.

Por otra parte esta necesidad de mejorar la financiación se extiende también, y de manera importante, a la Cooperativa de Trabajo Asociado que actúa en el tráfico económico y para la que resulta esencial. Dicha financiación requiere una reflexión detenida, dada la crítica que en la doctrina se mantiene sobre su insuficiencia y la incidencia negativa que la misma tiene en la marcha de la cooperativa ${ }^{15}$. Se ha dicho que estas entidades tienen una menor aproximación a las entidades financieras, bien porque sus dirigentes tengan una menor relación con éstas que la que se reconoce a las sociedades capitalistas, o una menor relación derivada sencillamente de su actuación más reducida en este ámbito financiero.

La legislación de cooperativas ha sido siempre celosa de la mayor garantía que debían ofrecer estas entidades, como si inicialmente admitiese una menor fiabilidad en relación con las sociedades capitalistas y una necesidad específica de consolidar este tipo social frente a sus proveedores y prestamistas. El art. 55 de la Ley General de Cooperativas establece en esta línea un fondo específico. Dice este precepto que «El Fondo de Reserva Obligatorio destinado a la consolidación, desarrollo y garantía de la cooperativa es irrepartible entre los socios». Y añade que «con independencia del Fondo de Reserva Obligatorio, la cooperativa deberá constituir y dotar los fondos que, por la normativa que le resulte de aplicación, se establezcan con carácter obligatorio en función de su actividad o calificación». En otras palabras, el Fondo de Reserva Obligatorio tiene un carácter adicional y tiene, entre otras finalidades, la garantía de la cooperativa frente a sus acreedores.

Desde nuestro punto de vista la necesidad de garantía de la cooperativa debe examinarse en relación con sus dos tipos de acreedores: proveedores y financiadores. En relación con los primeros, su garantía depende del comportamiento de la cooperativa misma. Se trata de un tráfico muy a corto plazo en el que la fiabilidad frente al acree-

15 Esta doctrina ha estado muy extendida y cuenta con una cierta tradición. Es conocida la opinión de VIDAL, I., en su artículo "La Economía Social y el Tercer Sector», Escuela Libre Editorial, 2003, págs. 946 y ss. Distingue la autora cuatro etapas: dificultad en el primer acercamiento a la entidad financiera, exigencia de mayores garantías, precios superiores a los de mercado y solicitud de una mayor cantidad de documentación. 
dor se gana (o se pierde) día a día. Plazo corto en el que el deudor se defiende mejor porque no puede acumular deudas importantes que a corto plazo no puede atender.

Mayores problemas ofrece la garantía a medio o largo plazo. Esta garantía externa normalmente al patrimonio de la propia cooperativa puede ser atendida con técnicas diversas. En esta búsqueda de nuevas figuras o replanteamiento de otras ya conocidas vuelve a plantearse la utilidad de los préstamos participativos ${ }^{16}$. Cabe indicar que estos préstamos requieren una dimensión mínima de la deuda que de sentido a la información, observaciones y participación de los prestamistas en la gestión, sin negar que la figura pueda ser de utilidad en determinados supuestos.

Otra garantía susceptible de ser tomada en consideración es la hipotecaria. Tanto la inmobiliaria como la mobiliaria (hipoteca de establecimiento mercantil y, en general, de los bienes comprendidos en la hipoteca mobiliaria). Es una figura que se utilizó en los préstamos concedidos a cooperativas por el antiguo Fondo de Protección del Trabajo. Se concedieron en circunstancias muy difíciles de crisis económica general y, específicamente, empresarial. Puede ser una figura que recupere su extensión en circunstancias de mercado normales.

De interés notable resulta igualmente la posibilidad y realidad de los préstamos concedidos por las cooperativas de crédito. Su aspecto positivo, a destacar en este punto, es el valor de la actividad crediticia de proximidad, dada su actuación en un territorio determinado. Todo ello con independencia de la consideración de socios que puedan tener las Cooperativas de Trabajo Asociado, lo que llevaría aún más a reforzar los vínculos prestamista-prestatario.

Otra alternativa que parece ganar terreno es la llamada Banca Ética, entre cuyos objetivos específicamente sociales deben de incluirse, en mi opinión, la figura de las Cooperativas de Trabajo Asociado. La característica de este tipo de banca es la financiación de proyectos sostenibles de diverso contenido, entre los cuales se incluye el desarrollo social.

Un modelo clásico es partir de la financiación bancaria con la incorporación de la garantía recíproca prestada a través de sociedades de esta misma denominación. En definitiva se trata de un fenómeno de mutualización de garantías, hoy de actualidad en la Unión Europea, donde gran parte de los países piden su establecimiento en relación

16 Véase a este respecto ROCAFORT NICOLAU, N., «La financiación cooperativa mediante la emisión de títulos participativos», en Revista de Economía Pública, Social y Cooperativa CIRIEC-España, agosto, 2010, págs. 141 y ss. 
con las emisiones de deuda soberana debido al alto coste que tiene su emisión hecha individualmente por determinados países miembros.

La principal ventaja de esta forma de garantía está en la técnica de mutualización, que supone contribuir con la aportación a la sociedad y desembolso de las participaciones sociales que suscriban, que será necesario para obtener una determinada garantía de la sociedad, y la constitución de un fondo social objetiva y subjetivamente amplio y cuyos servicios pueden ser utilizados por los aportantes en la forma señalada en los estatutos correspondientes. La Ley 1/1994, de 11 de marzo, que regula las sociedades de garantía recíproca a que nos referimos ${ }^{17}$, define las características de estas entidades: «las pequeñas y medianas empresas, con el fin de facilitar el acceso al crédito y servicios conexos, así como la mejora integral de sus condiciones financieras, podrán constituir sociedades recíprocas con capital variable, en las que los socios no responderán personalmente de las deudas sociales». Su objeto es el otorgamiento de garantías personales. A los socios se les exige su participación en el capital social en proporción al importe de las deudas cuya garantía solicite de la sociedad. El derecho de los socios, que aquí interesa, es solicitar las garantías y el asesoramiento de la sociedad dentro de los límites establecidos en los estatutos (art. 22). Su obligación es hacer las aportaciones correspondientes a las participaciones sociales que suscriban.

Las ventajas de estas sociedades y de esta técnica que aquí se indican son, principalmente, la obtención de una garantía que las entidades financieras considerarán normalmente como solvente y la obtención de un asesoramiento especializado en materia financiera.

Las sociedades de garantía recíproca permiten, además, la colaboración de las Administraciones a través de la figura de los socios protectores. Es evidente que su presencia debe facilitar la constitución de la sociedad y lo hará de manera importante, permitiendo igualmente el conocimiento de la realidad, dada su presencia en los órganos de la sociedad. Igualmente, facilitará la comparación entre el juego de esta figura del socio protector y el otorgamiento de subvenciones directas a las empresas. El hecho, por otro lado, de que las sociedades de garantía recíproca deban estar integradas por sociedades medias y pequeñas

17 En efecto dichas sociedades se encuentran reguladas en la Ley 1/1994, normativa a través de la cual se posibilita la integración y adaptación de la garantía en el sistema financiero, integrándose asimismo a las pymes dentro del sistema financiero al permitirles acceder a financiación en mejores condiciones de tipo de interés y plazo, con la consiguiente disminución de las necesidades de provisión y el consumo de recursos propios por parte de las entidades de crédito en función de la ponderación del aval. 
permite a las Administraciones cumplir el reiterado compromiso de los poderes públicos con las pymes en un campo muy importante para éstas como es la financiación.

Las sociedades de garantía recíproca abren paso (y demandan) una intensificación del movimiento asociativo empresarial y, por lo que aquí interesa, de las Cooperativas de Trabajo Asociado. La mutualización de garantías es, en definitiva, un movimiento asociativo que permite alcanzar lo que no se puede lograr individualmente. Los poderes públicos deben apoyar esta figura, no solo porque se inscribe en una exigencia constitucional, como ya vimos, sino porque descarga de presión al Sector Público y reduce el coste de la financiación en comparación con las subvenciones directas.

Con carácter general la Ley 14/2013, de 27 de septiembre, de apoyo a los emprendedores y su internacionalización, ha establecido unos mínimos en el capital y en los recursos propios de las sociedades de garantía recíproca que podría abocar a procesos de integración entre ellas, con la finalidad última de incrementar la actividad en favor de las pymes, siempre con el acuerdo de las partes involucradas en cada caso. A resultas de la citada modificación ha pasado a exigirse a estas sociedades un capital social mínimo de diez millones de euros y unos recursos propios no inferiores a 15 millones de euros.

Ahora bien sin perjuicio de lo anterior ha de reconocerse que, en la actualidad, las sociedades de garantía recíproca españolas, como entidades financieras de intermediación que son, se han visto afectadas por la caída del negocio de las empresas en este periodo de crisis, en el que se ha producido también un importante proceso de reestructuración en el sector bancario. Ello ha motivado una reducción del volumen de avales otorgados y el encarecimiento de los costes de intermediación.

En nuestra opinión estas sociedades deben orientarse a establecer un sistema de cooperación entre entidades financieras y sociedades de capital público-privado cuyo objetivo sea hacer fluir el crédito a pymes y autónomos. Ahora bien la actuación de estas entidades no debe limitarse únicamente a programas que permitan a las pymes obtener financiación en condiciones preferentes, sino también al establecimiento de sinergias con inversores que ofrezcan recursos alternativos a la financiación bancaria. En este sentido la adopción de garantías mediante avales de las sociedades de garantía recíproca (los cuales son a primer requerimiento, presentando además un carácter líquido, no requiriéndose en consecuencia dotación alguna de provisiones) y la implicación de inversores privados deben potenciarse y convertirse en complemento a la financiación tradicional para lograr 
impulsar el crédito al emprendimiento y consolidar el crecimiento de las pymes.

Se hace necesario además que el Ejecutivo, a través del Instituto de Crédito Oficial (ICO), así como a través de la propia Unión Europea, el Banco Europeo de Inversiones (BEI) u otros organismos faciliten el tránsito a un sistema financiero más desbancarizado en donde estas sociedades jueguen un papel mucho más importante. Debe pues tratar de potenciarse el sector desde el punto de vista financiero, para que pueda desempeñar un papel más activo en el proceso de recuperación del tejido empresarial, de acuerdo con la demanda de los propios empresarios y en sintonía con el perfil de este sector en los países de nuestro entorno.

\section{Reflexiones finales acerca de la formación y difusión del cooperativismo}

Tal y como se ha expuesto a lo largo de este trabajo las cooperativas de trabajo asociado sirven al desarrollo de políticas sociales y de adscripción de entidades que facilitan la creación de empleo estable, así como una mayor participación de los trabajadores. Su funcionamiento presenta un carácter democrático, siendo su régimen de libre adhesión y baja voluntaria, con un sistema de derechos y de participación de los socios muy amplio. Estas características contribuyen a que la aproximación de los trabajadores a la toma de decisiones en la empresa sea máxima, con un aumento de la productividad y de la responsabilidad y estabilidad de aquéllos, lo que constituye un hecho muy positivo que se inscribe en la mejor política de empleo posible.

Por su parte las cooperativas de viviendas y las de consumidores y usuarios contribuyen a la realización de políticas sociales relacionadas esencialmente con el consumo, siendo ejecutadas directamente por los interesados. A tal efecto el Capítulo III, Título I de la Constitución establece, entre otros, dos mandatos concretos que constituyen dos políticas sociales determinadas: el acceso de los ciudadanos a una vivienda digna y adecuada y el fomento de la defensa de los consumidores a través de su propia organización. Más concretamente, la Ley Fundamental establece las citadas políticas en los siguientes términos: "Todos los españoles tienen derecho a disfrutar de una vivienda digna y adecuada. Los poderes públicos promoverán las condiciones necesarias y establecerán las normas pertinentes para hacer efectivo este derecho (...)» (art. 47). Aquí el ordenamiento jurídico, junto a medidas muy diversas de promoción del acceso a la vivienda (de carácter administrativo y fiscal, princi- 
palmente) utiliza la figura jurídica de la cooperativa para permitir que los propios interesados puedan realizar esta política. El mismo cuadro se repite en relación con los consumidores. Sin perjuicio de numerosas normas y medidas de protección del consumo, la Constitución ordena a los poderes públicos que fomenten «sus organizaciones». También aquí se da un conjunto de normas y medidas diversas pero, además, se utilizan figuras y técnicas jurídicas ya establecidas por el ordenamiento para que los propios interesados puedan realizar estas políticas. Es la cooperativa de consumidores y usuarios. La nota diferencial de estas entidades se concreta pues en el hecho de que permiten que los propios ciudadanos realicen las políticas sociales que les interesan.

Ciertamente constituye un hecho innegable que las empresas de economía social tienen una mayor exposición a las dificultades de tesorería y capitalización. No pueden acceder a los mercados de capitales o lo hacen con gran dificultad y sus conexiones con los grupos financieros son muy escasas. Su financiación proviene, en primer lugar, de las aportaciones de los socios. Dentro de ellas tiene un particular interés teórico (y en algunos casos, práctico) la capitalización de las prestaciones de desempleo que debe percibir cada trabajador. Son pues dos ventajas: la capitalización, que permite su inversión de una vez, y la exención en el IRPF, que podría tener importancia al tratarse de un Impuesto progresivo.

La segunda fuente de financiación externa la constituyen las subvenciones dadas por las Administraciones y, muy principalmente por la Autonómica, para la creación de un puesto de trabajo o para la incorporación de nuevos socios a las cooperativas de trabajo asociado o sociedades laborales. Se trata de subvenciones reducidas que debían de ser mayores si se atendiese al mandato constitucional de fomentar las sociedades cooperativas y facilitar el acceso de los trabajadores a la propiedad de los medios de producción (art. 129.2 CE) pero que resultan de utilidad aunque ésta sea, como acabamos de decir, limitada. En definitiva, son cantidades que tienen un valor complementario. Esta baja cuantía impide, además, que puedan tacharse como ayudas de Estado. Desde nuestro punto de vista no lo son en ningún caso, ya que la Unión Europea tiene como uno de sus objetivos la cohesión social y las empresas asociativas llevan a cabo esta cohesión. La Unión Europea debería ser más cuidadosa en este campo si no quiere que su objetivo de cohesión sea poco más que una expresión retórica. De cualquier manera, muy difícilmente podrían adjetivarse estas subvenciones como ayudas de Estado, dado su grado de generalidad (muy amplio) y el hecho de que en última instancia les sería aplicable la cláusula «de minimis» que excluye las entregas de pequeña entidad. 
La cuestión de la capitalización de las empresas de economía social $y$, en definitiva, de su financiación, representa uno de los problemas más importantes que tiene planteados este sector empresarial. A tal efecto estimamos que habrían de ensayarse fórmulas de garantía colectiva que permitiesen aportaciones razonables y proporcionales y una reducción del riesgo por su carácter colectivo. Los pasos que se han dado en este campo a través de la creación de sociedades de garantía recíproca han dado un resultado satisfactorio y pienso que esta figura debe de ser uno de los referentes en la búsqueda de una mejor financiación. De cualquier manera, las posibilidades están abiertas para los trabajadores, las empresas, las Administraciones, las entidades rectoras de la economía social y las entidades financieras.

En la actualidad las necesidades existentes de adaptación al mercado (actualización de productos y servicios) vienen a poner de manifiesto la urgencia de articular mecanismos de colaboración entre las distintas sociedades cooperativas, en aras de alcanzar una unión de objetivos y de medios que permita llegar a donde no es posible hacerlo individualmente ${ }^{18}$. El ordenamiento jurídico español ofrece diversas formas y técnicas asociativas que constituyen la herramienta adecuada para llevar a cabo esta colaboración. La Ley 27/1999, de 16 de julio, de Cooperativas, regula las cooperativas de segundo grado, el grupo cooperativo, los consorcios y las uniones de este tipo de sociedades. Todas estas figuras facilitan la elección del tipo de colaboración adecuado para un objetivo determinado e incluso una actuación en común de carácter permanente.

En este sentido estimamos que el primer requisito para el desarrollo del cooperativismo lo constituye la información, posteriormente la formación de los trabajadores que quieran integrarse en él y, finalmente, la difusión del modelo mismo. La fuerza de los modelos de empresa capitalista privada y empresa pública es tan intensa que las sociedades cooperativas han pasado a constituir una excepción.

La formación ofrece a nuestro juicio un carácter esencial. Las cooperativas tienen a su favor el factor de la puesta en común por parte de los trabajadores de su propia experiencia. El primer paso para la investigación es la experiencia, entendida como el conocimiento científico de los hechos que generan un resultado y una situación. El ca-

18 Acerca de esta implementación de mecanismos de colaboración véase GADEA SOLER, E., "Crisis e intercooperación: las cooperativas de segundo o ulterior grado como instrumento de colaboración empresarial», Boletín de la Asociación Internacional de Derecho Cooperativo (Journal International Association of Cooperative Law), núm. 44, 2010, págs. 251 y ss. 
rácter de interesados propios o copropietarios que tienen los socios cooperativos facilita al máximo la puesta en común de la experiencia a que nos referimos y los avances en la investigación que sean procedentes. Nada externo se excluye de este proceso. Ni otras aportaciones, ni la aplicación de las tecnologías que sean convenientes. Conviene insistir en este proceso de acumulación de experiencias por parte de los trabajadores. A partir de él, puede iniciarse un avance de la investigación que interese y puede tener como resultado el inicio de procedimientos y acciones de innovación siempre esenciales en la actividad empresarial.

Este razonamiento, que es válido para la actividad económica que realice la cooperativa, lo es también para la organización y el desarrollo de la actividad social misma. La Ley General de Cooperativas de 1999 establece como uno de los Fondos sociales obligatorios el de Educación y Promoción. Sus actividades son muy amplias, aunque ceñidas lógicamente a su campo propio: formación en los principios y valores cooperativos, promoción cultural y asistencial y mejora de la calidad de vida. Nada impide que esta actividad de formación y promoción se dedique a otros campos, dada la amplitud de sus enunciados. Porque la propia Ley de 1999 permite que en el cumplimiento de estos fines las cooperativas puedan colaborar con otras sociedades y entidades pudiendo actuar en régimen de financiación común.

\section{Bibliografía}

Calvo Ortega, R., «Entidades de Economía Social: razones de una fiscalidad específica», en la obra colectiva (Dir.: Calvo Ortega, R.) Fiscalidad de las Entidades de Economía Social, Civitas, Madrid, 2005.

Cosín Ochalta, R., "Régimen de cooperativas y regímenes especiales», Carta Tributaria, núm. 2, 2006.

Crespo Miegimolle, M., Régimen especial de las sociedades cooperativas en el nuevo Impuesto sobre Sociedades, Comares, Granada, 1998.

De LuIS Esteban, J. M., «Reflexiones sobre el futuro fiscal de las cooperativas» y especialmente en el capítulo "La función social como contrapartida», en la obra colectiva (Dir.: Calvo Ortega, R.) Fiscalidad de las Entidades de Economía Social, Civitas, Madrid, 2005.

Del Arco Álvarez, J. L., Régimen fiscal de las cooperativas, 1969.

Gadea Soler, E., «Universidad y cooperativismo. Delimitación del concepto de cooperativa en una sociedad democrática avanzada: referencia a los principios cooperativos y a su discutida vigencia», Boletín de la Asociación Internacional de Derecho Cooperativo (Journal International Association of Cooperative Law), núm. 42, 2008. 
- «Crisis e intercooperación: las cooperativas de segundo o ulterior grado como instrumento de colaboración empresarial», Boletín de la Asociación Internacional de Derecho Cooperativo (Journal International Association of Cooperative Law), núm. 44, 2010.

- «Cooperativismo de consumo: la adaptación del modelo a las nuevas condiciones del mercado», Boletín de la Asociación Internacional de Derecho Cooperativo (Journal International Association of Cooperative Law), núm. 45, 2011.

Gadea Soler, E. y Díaz Ácimas, L. A., Régimen jurídico de las cooperativas de Cantabria: análisis de la Ley 6/2013, de 6 de noviembre, Dykinson, Madrid, 2014.

Martín Fernández, J., Martín Salcines, F. y Rodríguez Márquez, J., Cuestiones tributarias y contables de las cooperativas, lustel, 2006.

Monzón, J. L., «Economía Social y Sociedad de Bienestar: actores y ámbitos de actuación en España», en Anuario de la Economía Social 2007-2008, Cepes, Madrid, 2008.

ROCAFORT NiCOLAU, N., «La financiación cooperativa mediante la emisión de títulos participativos», en Revista de Economía Pública, Social y Cooperativa CIRIEC-España, agosto, 2010.

Rodrigo Uría, Aurelio Menéndez y Mercedes Vérgez, Curso de Derecho Mercantil, vol. I, Civitas, Madrid, 1999.

VIDAL, I., «La Economía Social y el Tercer Sector», Escuela Libre Editorial, 2003. 